\title{
Anterior spinal artery functioning as a dominant anastomotic channel to the vertebrobasilar system
}

\author{
Hidetsugu MAEKAWA Michihiro TANAKA \\ Department of Neurosurgery, Kameda Medical Center
}

Key words

anterior spinal artery, collateral circulation, moyamoya disease

< Correspondence Address: Hidetsugu MAEKAWA, 929 Higashi-cho, Kamogawa, Chiba, 296-8602, Japan E-mail: h-maekawa@kameda.jp > (Received May 1, 2014 : Accepted July 4, 2014)

doi: 10.5797/jnet.ioi.2014-0010

The anterior spinal artery (ASA) is the blood vessel that supplies the anterior spinal cord, but rarely serves as a collateral to the vertebrobasilar system. This report describes a rare case of bilateral vertebral artery (VA) occlusion associated with moyamoya disease in which the ASA functioned as a dominant anastomosis to the vertebrobasilar system. The case report is followed by a discussion of the significance of the development of the collateral circulation via the ASA.

\section{Case report}

A 37-year-old man with known moyamoya disease and renal failure developed hypesthesia of the right arm. MRI of the cervical cord demonstrated a significantly dilated and tortuous flow void structure on the anterior surface of the cervical spinal cord, which was suspected to be an arteriovenous shunt. On coronal view, the flow void was revealed as a single continuous vessel (Fig. 1). The proximal intracranial portions of the bilateral vertebral arteries were not shown on the head MRA. Instead, a tortuous vessel seen as a flow void on MRI of the cervical cord was depicted (Fig. 2A). The supraclinoid portion of both internal carotid arteries was nearly occluded, as is the typical radiological appearance of moyamoya disease. The blood flow in the bilateral middle cerebral arteries seemed to arise dominantly from the basilar artery via the posterior communicating arteries (Fig. 2B). On MRA of the neck, the signal of the right VA disappeared at the C2 level, and the signal of the left VA disappeared at the junction of the V3 and V4 portion. On the other hand, the radiculomedullary artery arising from the right cervical VA at the C6/7 level connected to the dilated and tortuous ASA running upward to the VA (Fig. 2C). Dilated veins around the cervical spinal cord were not detectable, including the original source images. Based on these findings, we confirmed that the tortuous flow void structure was the ASA itself and that it functioned as a dominant collateral vessel to the vertebrobasilar system. The patient was conservatively treated without performing cerebral angiography or any surgical or endovascular intervention.

\section{Discussion}

The ASA runs anterior to the spinal cord from the level of the medulla oblongata down to the conus medullaris. The artery is supplied by the radiculomedullary arteries arising from the segmental arteries. These segmental arteries originate from the VA and the deep cervical or ascending cervical artery. ${ }^{1)}$

Some cases have been reported in which the ASA anastomoses to the vertebrobasilar system as a result 


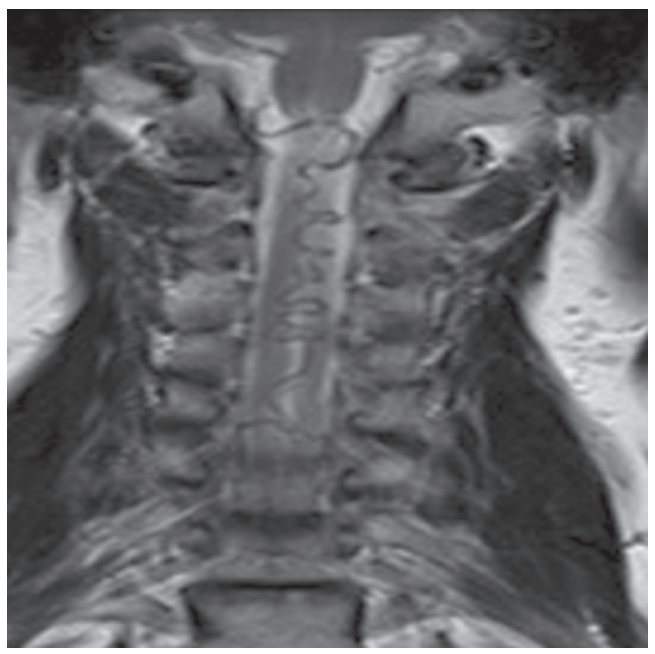

Fig. 1

T2-weighted cervical MRI, coronal view.

Note a continuous and tortuous flow void on the anterior surface of the cervical cord and medulla oblongata.

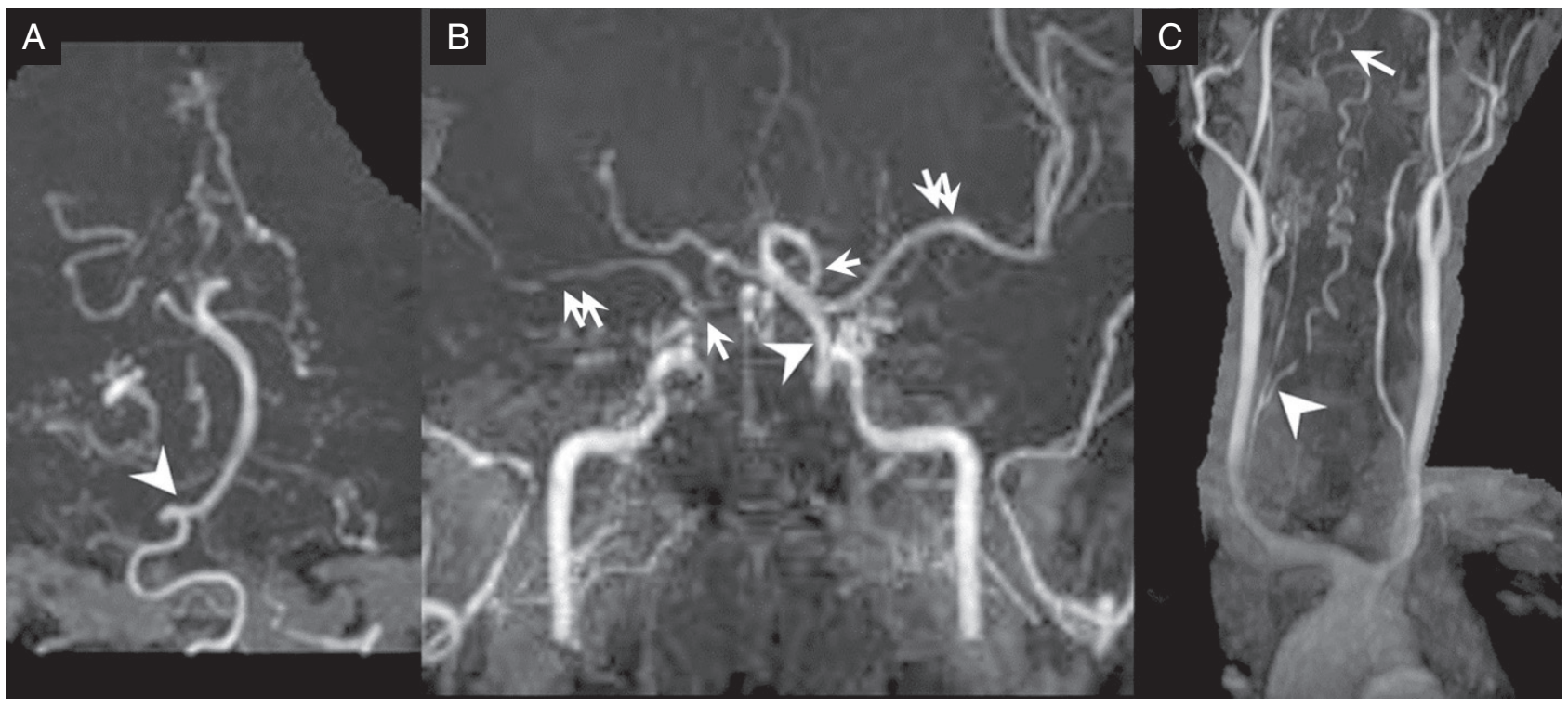

Fig. 2

A, B: Head MRA, C: Neck MRA. All images are in anterior-posterior view.

A: The proximal intracranial part of the bilateral vertebral arteries is not depicted. Arrowhead indicates the origin of the anterior spinal artery from the vertebral artery.

B: The signal of most distal part of both internal carotid arteries disappears. The bilateral middle cerebral arteries (paired arrows) are supplied dominantly by the basilar artery (arrowhead) via the posterior communicating arteries (arrows).

$\mathrm{C}$ : The tortuous artery connecting to the basilar artery is confirmed as a dilated anterior spinal artery originating from the right vertebral artery at the C6/7 level on neck MRA (arrowhead). Arrow indicates the identical point of the arrowhead in Fig. 2A, where the anterior spinal artery connects to the vertebral artery. 
of occlusion of the bilateral VAs associated with acute ischemic stroke or moyamoya disease. ${ }^{2-5)}$ Although this type of collateral circulation is rare, the ASA is a potential anastomotic route to the vertebrovasilar system.

The most cranial part of the ASA originates from the $\mathrm{VA}$, and the direction of the blood flow in the ASA is from cranial to caudal in the upper cervical level. ${ }^{6}$ In cases of bilateral VA occlusion without sufficient collateral circulation, the blood flow of the vertebrobasilar system decreases markedly. The flow direction in the ASA depends on the equilibrium of perfusion pressure between the vertebrobasilar system and the ASA. Therefore, if the blood pressure of the intracranial vertebrobasilar system decreases due to a steno-occlusive lesion in the VA, blood flow in the ASA may be towards the cranial direction.

We speculate that the prominent and tortuous ASA filling the vertebrobasilar system indicates chronic hypoperfusion of the global brain rather than that of the posterior fossa. As in previously reported cases with moyamoya disease, the bilateral VA and the internal carotid artery were affected in our case. The distal part of the bilateral internal carotid arteries was not opacified, and the bilateral middle cerebral arteries were supplied dominantly by the basilar artery. Similar findings were observed in previous cases. ${ }^{4)}$ The dilatation and meandering of the ASA results from the long-standing increased blood flow to supply blood to not only the vertebrobasilar system but also the cerebral hemispheres.

In conclusion, the ASA may serve as an anastomotic channel when the blood flow of the vertebrobasilar system decreases critically and when the collateral circulation is poor. This finding may indicate chronic hypoperfusion in the area of posterior fossa and also in the entire brain.

The authors declare that they have no conflict of interest.

\section{References}

1) Lasjaunias P BA, terBrugge KG. Spinal and spinal cord arteries and veins, in Surgical Neuroangiography. vol. 1. 2nd ed, Berlin, Springer, 2001, 73-164.

2) Kang HS, Han MH, Kim SH, et al. Anterior spinal artery as a collateral channel in cases of bilateral vertebral arterial steno-occlusive diseases. AJNR Am J Neuroradiol 2007; 28: 222-225.

3) Yamakawa H, Yoshimura S, Iwama T. Anterior spinal artery as a collateral channel in patients with acute bilateral vertebral artery occlusions. Two case reports. Neurol Med Chir (Tokyo) 2009; 49: 354-358.

4) Karasawa J, Kikuchi H, Furuse S, et al. Enlarged anterior spinal artery as collateral circulation. J Neurosurg 1974; 41: 356-359.

5) Miyamoto S, Kikuchi H, Karasawa J, et al. Moyamoya disease associated with bilateral occlusion of the vertebral artery. Surg Neurol 1984; 22: 21-28.

6) Di Chiro G, Fried LC. Blood flow currents in spinal cord arteries. Neurology 1971; 21: 1088-1096. 